 \\ Preprints are preliminary reports that have not undergone peer review. They should not be considered conclusive, used to inform clinical practice, or referenced by the media as validated information.
}

\section{Characterization of the vaginal microbiota of women with premature ovarian insufficiency}

\section{Jingyi Wen}

Tongji Hospital of Tongji Medical College of Huazhong University of Science and Technology

\section{Yanzhi Feng}

Tongji Hospital of Tongji Medical College of Huazhong University of Science and Technology

\section{Suzhen Yuan}

Tongji Hospital of Tongji Medical College of Huazhong University of Science and Technology

\section{Wei Yan}

Tongji Hospital of Tongji Medical College of Huazhong University of Science and Technology

Jinjin Zhang

Tongji Hospital of Tongji Medical College of Huazhong University of Science and Technology

Aiyue Luo

Tongji Hospital of Tongji Medical College of Huazhong University of Science and Technology

Shixuan Wang ( $\nabla$ shixuanwang@tjh.tjmu.edu.cn )

Tongji Hospital of Tongji Medical College of Huazhong University of Science and Technology https://orcid.org/0000-0002-8610-952X

\section{Research}

Keywords: Premature ovarian insufficiency, Vaginal microbiota, 16S rRNA gene sequencing , Ovarian reserve, Ovarian function

Posted Date: May 5th, 2021

DOI: https://doi.org/10.21203/rs.3.rs-439533/v1

License: (c) (i) This work is licensed under a Creative Commons Attribution 4.0 International License. Read Full License 


\section{Title page}

2 Title:

3 Characterization of the vaginal microbiota of women with premature ovarian insufficiency

\section{Authors' full name}

5 Jingyi Wen ${ }^{1,2,3}$, Yanzhi Feng ${ }^{1,2,3}$, Suzhen Yuan ${ }^{1,2,3}$, Wei Yan ${ }^{1,2,3}$, Jinjin Zhang ${ }^{1,2,3 \#}$, Aiyue

$6 \quad$ Luo $^{1,2,3 \#}$, Shixuan Wang ${ }^{1,2,3 \#}$

$7 \quad{ }^{\#}$ Corresponding to Shixuan Wang, Aiyue Luo and Jinjin Zhang, Fax: 86(27)83663078, Tel:

8 86(27)83663078, E-mail: shixuanwang@tjh.tjmu.edu.cn, 691135685@qq.com,

9 jinjinzhang@tjh.tjmu.edu.cn.

\section{Authors' institutional affiliations}

$11{ }^{1}$ Department of Obstetrics and Gynecology, Tongji Hospital, Tongji Medical College,

12 Huazhong University of Science and Technology, 1095 Jiefang Avenue, Wuhan, Hubei,

13 China.

$14{ }^{2}$ National Clinical Research Center for Obstetrical and Gynecological Diseases, Tongji

15 Hospital, Tongji Medical College, Huazhong University of Science and Technology, 1095

16 Jiefang Avenue, Wuhan, Hubei, China.

$17{ }^{3}$ Cancer Biology Research Center, Key Laboratory of the Ministry of Education, Tongji

18 Hospital, Tongji Medical College, Huazhong University of Science and Technology, 1095

19 Jiefang Avenue, Wuhan, Hubei, China. 
21 Jingyi Wen: jingyiwen@tjh.tjmu.edu.cn

22 Yanzhi Feng: 13265096548@163.com

23 Suzhen Yuan: 494723018@qq.com

24 Wei Yan: 470877220@qq.com

25 Jingjin Zhang: jinjinzhang@ tjh.tjmu.edu.cn

26 Aiyue Luo: 691135685@qq.com

27 Shixuan Wang: shixuanwang@ tjh.tjmu.edu.cn.

28

29

30

31

32

33

34 
Background: Premature ovarian insufficiency (POI) is a complex reproductive endocrine disease that can affect multiple systems. It is highly heterogeneous in etiology and the exact etiology remains unclear. Infectious factors may be related to POI, but researches on the alterations of microbiome in POI patients are scarce.

Results: Vaginal swabs were collected from 52 POI patients (29 for sequencing, 23 for validation) and 46 healthy individuals of comparable age (26 for sequencing, 20 for validation). 16S rRNA gene sequencing targeting the V3-V4 hypervariable regions was performed to evaluate the alterations of vaginal microbiota in POI patients. The relative abundance of Actinobacteria (23.34\% vs $10.65 \%, \mathrm{P}=0.017)$, Atopobium ( $11.11 \%$ vs $0.01 \%$, P $<0.001$ ), and Gardnerella ( $8.05 \%$ vs $3.14 \%, \mathrm{P}=0.002)$ were significantly increased in POI patients, while Bifidobacterium (3.95\% vs $7.44 \%, \mathrm{P}=0.017$ ) was significantly decreased. Cluster analysis of dominant strains showed that the proportion of POI patients whose predominant bacteria were not Lactobacillus was increased than the control group, but the difference was not statistically significant $(8 / 29$ vs $2 / 26, P=0.105)$. More interestingly, these changes in vaginal microbiota were significantly correlated with declined ovarian function in POI patients, including decreased ovarian reserve, ovarian endocrine disruption, and symptoms of perimenopausal syndrome. Actinobacteria, Atopobium, and Gardnerella appeared to be detrimental to ovarian function, while Bifidobacterium seemed to be benificial.

Conclusions: The present study revealed the correlation between vaginal microbiota and POI, fill the gap in the field of microbial and POI research and provide a new research strategy for POI. But the causal relationship is still unclear, and we could not clarify detailed roles of specific constituents of the vaginal microbiota in the pathogenesis of POI. Therefore, future studies are needed to explore potential mechanisms underlying the association of vaginal microbiota and POI. 
60 Keywords: Premature ovarian insufficiency, Vaginal microbiota, 16S rRNA gene sequencing,

61 Ovarian reserve, Ovarian function.

62

63

64

65

66

67

68

69

70

71

72

73

74

75 
77 Premature ovarian insufficiency (POI) is a clinical syndrome defined by loss of ovarian activity before 40 years old. Clinically, POI is characterized by menstrual disturbance (oligomenorrhea or amenorrhea) with raised gonadotrophins and low estradiol ${ }^{1}$. According to the epidemiological data, the prevalence of $\mathrm{POI}$ is approximately $1 \%$, population characteristics such as ethnicity may affect the prevalence ${ }^{1-3}$. Recently, it is believed that the morbidity is increasing, the reported morbidity may be lower than it actually is, and the age of onset tends to be younger. There is no effective method to predict the occurrence of POI now, and when patients reach the diagnostic criteria, their ovarian function is often nearly complete failure.

POI is a complex disease that can affect multiple systems. The clinical manifestation differs between individuals, mainly including menstrual disturbance, infertility and symptoms of perimenopausal syndrome ${ }^{1}$. Besides, POI patients may experience complications such as cardiovascular disease and osteoporosis, mainly due to the lack of estrogen ${ }^{4,5}$. A diagnosis of POI also has a significant negative impact on psychological wellbeing and quality of life $\mathrm{e}^{1,6}$.

91 Therefore, early prevention, early detection and early intervention to reduce the risk of 92 long-term complications and other systemically related diseases of POI are very important. 93 The clinically recommended treatments are hormone replacement therapy (HRT) and in vitro 94 fertilization-embryo transfer (IVF-ET) with eggs donation based on HRT ${ }^{1}$. However, for those 95 with HRT contraindication or no source of donated eggs, they often suffer from the disease 96 due to the absence of effective alternative therapies. POI is highly heterogeneous in etiology and the exact etiology remains unclear. Although a wide spectrum of causes has been considered, including genetic ${ }^{2,7-9}$, autoimmune ${ }^{3,10}$,

99 iatrogenic ${ }^{11-13}$ or environmental pollutants ${ }^{14,15}$. However, as many as half of patients are 100 diagnosed with idiopathic POI because the causal factors are unknown. The complexity of 
101 POI, the increasing morbidity, the decreasing age of onset, and the lacking of early warning

102 and treatment methods all indicate that it is of great importance to study POI.

103 The vaginal microbiota accounts for about $9 \%$ of the total human microbiota and remains

104 under-studied despite its importance for female health and future generations ${ }^{16-19}$. The bacteria

105 inhabiting the human vagina are thought to be the first line of defense against vaginal

106 infection as a result of both the competitive exclusion and direct killing of other, pathogenic

107 microbes ${ }^{16,20,21}$. Disruptions of normal vaginal microbiota have long been linked to pelvic

108 inflammatory disease $e^{22}$, miscarriages ${ }^{23}$, and prematurity ${ }^{24,25}$. The composition of vaginal

109 microbiota is quite dynamic, it is influenced to a large extend by factors like age, ethnicity,

110 genetics, sexual activity, diet, hygiene status, and gynecological or reproductive status of

111 women ${ }^{17,20,26-28}$. Apart from these, the previous studies have also found considerable changes

112 in the vaginal microbiota of postmenopausal women ${ }^{29-32}$. Lactobacilli is the dominant bacteria

113 in the vaginal of most women, while it dramatically decreases in postmenopausal women,

114 with the increase of non-lactic acid-producing bacteria, and the diversity of vaginal

115 microbiota also increases ${ }^{32}$. Changes in vaginal microbiota lead to reduced lactic acid

116 production and an increasing in the secretion of inflammatory factors such as tumor necrosis

117 factor (TNF)- $\alpha$, interleukin (IL)-6, and IL-8, which activate the immune system and cause the

118 body to be in a chronic inflammatory state ${ }^{33-36}$. These findings suggest that vaginal microbiota

119 is very important for maintaining female reproductive health and there is a relationship

120 between vaginal microbiota and ovarian function.

121 Menopause is a sign of ovarian aging. As a pathological ovarian aging, the reproductive

122 endocrine status of POI patients is similar to that of postmenopausal women ${ }^{1,37}$, while

123 whether their vaginal microbiota will undergo similar changes is still unknown and remains to

124 be explored. In addition, inflammation and autoimmunity are important factors that affect

125 ovarian function and promote the occurrence and development of $\mathrm{POI}^{3,38}$. Moreover,

126 cross-sectional studies have also found that the levels of TNF- $\alpha$, IL- $1 \alpha$, IL-1 $\beta$, IL-6 in the

127 serum and (or) follicular fluid of POI patients are significantly higher than those in healthy 
128 controls $^{39,40}$. And the levels of these cytokines have significant correlations with sex hormones.

129 Considering the important role of vaginal microbiota in maintaining female reproductive

130 health, as well as the potential connection between POI and chronic inflammation caused by

131 changes of vaginal microbiota, it is of great importance to explore the change pattern of

132 vaginal microbiota in POI patients.

133 The purpose of this case-control study was to draw the characteristic spectrum of vaginal

134 microbiota in POI patients by comparing with healthy women of comparable age, and to

135 analyze the correlation between changes in vaginal microbiota and the declined ovarian

136 function. Through this research, we want to lay a foundation for further in-depth exploration

137 of the etiology and pathogenesis of POI, and provide new research strategies for early

138 detection and treatment of POI.

\section{Methods}

\section{Study population and sample collection}

141 This was a case-control study to describe the characterization of the vaginal microbiota of

142 POI patients. POI is defined as oligo/amenorrhea for at least 4 months before 40 years old,

143 and an elevated follicle stimulating hormone (FSH) level $>25$ IU/L on two occasions $>4$

144 weeks apart ${ }^{1}$. Women younger than 40 years old with regular menstruation and normal

145 ovarian function were included as the control group. Exclusion criteria were as follows: (1)

146 women during pregnancy or lactation, (2) women who have taken exogenous sex hormones in

147 the past three months, (3) women who have received antibiotics, probiotics, prebiotics,

148 intravaginal drugs, etc. in the last month, (4) women who are suffering from serious organic

149 diseases and requiring long-term medical treatment, (5) women who are participating in other

150 clinical trials. 
151 This study was approved by the Ethical Committee of Tongji Hospital, Tongji Medical

152 College, Huazhong University of Science and Technology. All participants agreed to join the

153 study and signed an informed consent form. From April 2018 to May 2019, a total of 52

154 eligible POI patients and 46 controls were included in the Menopause \& Ovarian Aging

155 Specialty Clinic of Tongji Hospital. Participants' basic clinical characteristics data were

156 obtained from questionnaires and electronic medical records by experienced interviewers. The

157 information included in the analysis included demographic characteristics, lifestyle,

158 reproductive history, menstrual history, serum basal sex hormone levels (days 2-5 of the

159 menstrual cycle for controls), Kupperman index (KMI) scale, Hospital anxiety and depression

160 (HAD) scale.

161 Vaginal swab samples were collected for each participant by experienced gynecologists. All

162 samples were obtained in compliance with clinical ethics regulations, and the principle of

163 sterility was strictly observed during the sampling process. Samples from 29 POI patients and

16426 controls were used for 16S rRNA gene sequencing, while samples from the other 23 POI

165 patients and 20 controls were used to verify the sequencing results by quantitative

166 polymerasechain reaction (qPCR). All samples were stored at $-80^{\circ} \mathrm{C}$ until assayed. Specimens

167 from all participants were processed similarly in terms of sample collection, storage, DNA

168 extraction, library preparation and sequencing.

\section{DNA extractions}

170 Genomic DNA was extracted from archived vaginal swab specimens using the E.Z.N.A.

$171{ }^{\circledR}$ Stool DNA Kit (D4015, Omega, Inc., USA) according to the manufacturer's instructions.

172 The reagent which was designed to uncover DNA from trace amounts of sample has been

173 shown to be effective for the preparation of DNA of most bacteria. Nuclear-free water was

174 used for blank. The total DNA was eluted in $50 \mu \mathrm{l}$ of elution buffer and stored at $-80^{\circ} \mathrm{C}$ until

175 measurement in the PCR by LC-Bio Technology Co., Ltd, Hang Zhou, Zhejiang Province,

176 China. 
178 The V3-V4 hypervariable regions of the bacterial small-subunit (16S) rRNA gene was

179 amplified with slightly modified versions of primers $341 \mathrm{~F}$

180 (5'-CCTACGGGNGGCWGCAG-3') and 805R (5'-GACTACHVGGGTATCTAATCC-3' $)^{41}$.

181 The 5 ' ends of the primers were tagged with specific barcodes per sample and sequencing

182 universal primers. PCR amplification was performed in a total volume of $25 \mu$ l reaction

183 mixture containing $50 \mathrm{ng}$ of template DNA, $12.5 \mu \mathrm{l}$ PCR premix, $2.5 \mu \mathrm{l}$ of each primer, and

184 PCR-grade water to adjust the volume. The PCR conditions to amplify the bacterial $16 \mathrm{~S}$

185 fragments consisted of an initial denaturation at $98^{\circ} \mathrm{C}$ for 30 seconds, 32 cycles of

186 denaturation at $98^{\circ} \mathrm{C}$ for 10 seconds, annealing at $54^{\circ} \mathrm{C}$ for 30 seconds, and extension at $72^{\circ} \mathrm{C}$

187 for 45 seconds, and then a final extension at $72^{\circ} \mathrm{C}$ for 10 minutes. The PCR products were

188 confirmed with 2\% agarose gel electrophoresis. Throughout the DNA extraction process,

189 ultrapure water, instead of a sample solution, was used to exclude the possibility of

190 false-positive PCR results as a negative control. The PCR products were purified by AMPure

191 XT beads (Beckman Coulter Genomics, Danvers, MA, USA) and quantified by Qubit

192 (Invitrogen, USA). The amplicon pools were assessed on Agilent 2100 Bioanalyzer (Agilent,

193 USA) and with the Library Quantification Kit for Illumina (Kapa Biosciences, Woburn, MA,

194 USA), respectively. The libraries were sequenced on NovaSeq PE250 runs platform.

195 Bioinformatics analysis

196 Samples were sequenced on an Illumina NovaSeq platform according to the manufacturer's

197 recommendations, provided by LC-Bio. Paired-end reads was assigned to samples based on

198 their unique barcode and truncated by cutting off the barcode and primer sequence.

199 Paired-end reads were merged using FLASH (v1.2.8) ${ }^{42}$. Quality filtering on the raw tags were

200 performed under specific filtering conditions to obtain the high-quality clean tags according

201 to the fqtrim (v0.94). Chimeric sequences were filtered using Vsearch software (v2.3.4) ${ }^{43}$.

202 After dereplication using DADA2, we obtained feature table and feature sequence ${ }^{44,45}$. Alpha 
203 diversity and beta diversity were calculated by normalized to the same sequences randomly.

204 Then according to SILVA (release 132) classifier, feature abundance was normalized using

205 relative abundance of each sample.

206 Alpha diversity was applied in analyzing complexity of species diversity for a sample through

207 five indices, including Observed-otus, Chao1, Shannon, Simpson, Goods_coverage, and all

208 these indices in our samples were calculated with QIIME $2^{46}$. The Shannon and Simpson

209 indices provide a quantitative measure of species diversity (richness and evenness), whereas

210 the Observed-otus and Chao1 indices provide a quantitative measure of species richness.

211 Goods_coverage index refers to the microbial coverage and actually reflects whether the

212 sequencing results represent the true situation of the sample. Beta diversity were evaluated by

213 QIIME2, the graphs were drawn by R package. Blast was used for sequence alignment, and

214 the feature sequences were annotated with SILVA (release 132) and NT-16S database for each

215 representative sequence. A cluster analysis and a LEfSe analysis were conducted to compare

216 differences in the composition of vaginal microbiota. Other diagrams were implemented using

217 the R package (v3.5.2). Bioinformatic analysis was performed using the OmicStudio tools at

218 http://www.omicstudio.cn/tool.

\section{Quantitative PCR and data analysis}

220 Quantitative PCR reactions were performed with Power SYBR Green PCR Master Mix

221 (Applied Biosystems, Foster City, California, USA) in samples from the validation population

222 to further verify the results of $16 \mathrm{~S}$ rRNA gene sequencing. All qPCR reactions contained 10

$223 \mu \mathrm{l}$ of $2 \times$ SYBR Green Master Mix, $2 \mu \mathrm{l}$ of primers (final concentration $10 \mathrm{pm} / \mu \mathrm{l}$ ), $500 \mathrm{ng}$ of

224 the DNA template. The primer sequences were listed in Supplementary table 1. The thermal

225 cycling conditions were as follows: an initial denaturation step at $95^{\circ} \mathrm{C}$ for $3 \mathrm{~min}$ followed by

22640 cycles of denaturation at $95^{\circ} \mathrm{C}$ for $15 \mathrm{~s}$, primer annealing at $65^{\circ} \mathrm{C}$ for $30 \mathrm{~s}$, extension at

$22772^{\circ} \mathrm{C}$ for $30 \mathrm{~s}$, and a fluorescence acquisition step at $72^{\circ} \mathrm{C}$ for $10 \mathrm{~min}$. DNAs extracted from

228 vaginal samples were amplified in triplicate, and the mean values were used for statistical 
analysis. Amplification and detection were performed on an ABI 7900HT Fast Real Time

230 PCR system (Applied Biosystems). The delta threshod cycle $(\Delta \mathrm{Ct})$ value was calculated and

231 used to compare the differences between groups in the abundance of the flora. The higher the

$232 \Delta \mathrm{Ct}$ value, the lower the abundance of the flora in the sample.

\section{Statistical analysis}

234 Shapiro-Wilk test was conducted to test the distribution types of continuous variables.

235 Continuous variables conforming to normal distribution were presented as mean $\pm \mathrm{SD}$

236 (standard deviation) and compared by group using the Student's $t$ test, while variables with

237 skew distribution were expressed as median (interquartile, IQR) or median (range) and

238 compared using the nonparametric Wilcoxon rank sum test. Categorical variables were

239 expressed as frequencies (percentages, \%) and compared using the chi-square test. KMI

240 scores ranging from $0-6,7-15$, and $>16$ were used to rate the degree of severity as none, mild

241 and moderate to severe, respectively ${ }^{47}$. HAD scores ranging from 0-7, and $>7$ represented

242 normal and anxiety/depression, respectively ${ }^{48}$. Spearman correlation was used to analyze the

243 correlation between vaginal microbiota and clinical indicators related to ovarian reserve,

244 ovarian endocrine function, and symptoms of perimenopausal syndrome. Meanwhile, for each

245 important phylum or genus with significant differences, we constructed the received operating

246 characteristic (ROC) curve and computed the area under the curve (AUC) value. A two-tailed

$247 P<0.05$ was considered statistically significant. All data were analyzed using IBM SPSS

248 Statistics 20.0 (SPSS Inc., Chicago, IL, USA).

\section{Results}

\section{Characteristics of the study population}

251 The age range of the 55 participants (29 POI patients, 26 controls) whose samples were used

252 for 16S rRNA gene sequencing was 17-39 years old. Among POI patients, the median age 
254 the controls, the median age was 33 (IQR: 28.75-38.25), the mean BMI was 20.89 \pm 2.39 .

255 There were no significant differences between the two groups in age and BMI, as did other

256 demographic characteristics, lifestyle, reproductive history, and HAD scores $(P>0.05)$

257 (Table1, Supplementary table 2). Whereas, the age of menarche in the POI group was

258 significantly lower than that of the controls $(P=0.022)$. Moreover, FSH and luteinizing

259 hormone $(\mathrm{LH})$ were significantly higher in the POI group $(P<0.001)$. Serum levels of

260 testosterone (T), estradiol (E2), anti-mullerian hormone (AMH), and inhibin B were lower

261 among POI patients than the controls $(P<0.001, \mathrm{P}=0.001, P<0.001, P<0.001$, respectively).

262 Levels of prolactin (PRL) and progesterone (PRG) were not significantly different between

263 the two groups $(P>0.05)$. These differences were basically in line with the clinical phenotype

264 and pathophysiological characteristics of POI.

Differences in the vaginal microbiota diversity between POI patients and the controls

266 No significant difference was found in the alpha diversity of vaginal microbiota between POI

267 patients and the controls (Supplementary Fig. 1 A-E). Whereas, beta diversity was

268 significantly different between the two groups according to the Anosim (analysis of

269 similarities, $R=0.083, P=0.01$ ) and showed in PCA (principal component analysis) plots

270 (Supplementary Fig. 1F).

271 Alterations in the composition of vaginal microbiota in POI patients

272 The dominant vaginal bacteria in both POI patients and the controls were Firmucutes at the

273 phylum level and Lactobacillus at the genus level (Supplementary Fig. 2A, E). The top five

274 bacterial genera in relative abundance in all samples were Lactobacillus, Atopobium,

275 Gardnerella, Streptococcus, and Bifidobacterium, respectively belonging to Firmucutes and

276 Actinobacteria (Fig. 1A). Moreover, at the phylum level, POI patients had a significantly

277 higher abundance of Actinobacteria (23.34\% vs $10.65 \%, P=0.017$ ), while the abundance of

278 Firmucutes was lower, but it was not statistically significant $(72.34 \%$ vs $83.99 \%, P=0.162)$ 
(Fig. 1B). At the genus level, the relative abundance of Atopobium and Gardnerella were

280 significantly increased in POI patients $(11.11 \%$ vs $0.01 \%, P<0.001 ; 8.05 \%$ vs $3.14 \%$,

$281 P=0.002$ ), while the relative abundance of Bifidobacterium was significantly decreased

282 (3.95\% vs 7.44\%, $P=0.017)$. The relative abundance of Lactobacillus was decreased in POI

283 patients but showed no significant difference between the two groups (62.98\% vs $79.54 \%$,

$284 P=0.08)$ (Fig. 1C).

285 At the species level, hierarchical clustering of bacterial community composition data showed

286 that the 55 vaginal samples could be assigned to six distinct clusters based on differences in

287 the composition of relative abundances of bacterial taxa (Fig. 2A). The average proportion of

288 Lactobacillus crispatus in cluster A $(N=15)$ was $95.89 \%$ (95\% CI: 93.92\%, 97.86\%), while

289 the average proportion of Lactobacillus iners in cluster B $(N=16)$ was $92.09 \%$ (95\%CI:

$29087.83 \%, 96.35 \%)$, and that of Lactobacillus sp. L-YJ in cluster C (N=8) was $54.47 \%(95 \% \mathrm{CI}$ :

$29146.33 \%, 62.61 \%)$. The communities in cluster $\mathrm{D}(N=2)$ and $\mathrm{E}(N=4)$ were dominated by

292 uncultured Lactobacillus sp. and Bifidobacterium, respectively. Finally, communities in

293 cluster $\mathrm{F}(N=10)$ exhibited greater diversity and evenness and included several codominant

294 taxa, including Atopobium, Streptococcus, Gardnerella, and Haemophilus. Lactobacillus was

295 still the dominant strain in most of the population (19/29 in the POI group, 22/26 in the

296 control group), while the proportion of cluster F in the POI group (8/29) was higher than that

297 in the control group (2/26), but the differences were both not statistically significant $(P=0.119$,

$298 P=0.105)$ (Fig. 2B).

299 To identify the specific and key bacterial taxa associated with POI, LEfSe analysis was

300 performed to generate the cladogram of the vaginal microbiota (Fig. 3B). At the phylum level,

301 Actinobacteria was significantly enriched in POI patients (Fig. 3A). At the genus level,

302 Atopobium and Gardnerella were dramatically enriched in POI patients, whereas

303 Bifidobacterium was significantly enriched in the controls. According to the LEfSe analysis,

304 these were all key phylotypes differentiating vaginal microbiota of POI patients and controls,

305 and might be potential microbiological markers for discriminating patients with POI. 
306 Collectively, these differences in the variation of vaginal microbial composition revealed a

307 correlation between vaginal microbiota and POI.

\section{Identification of key bacterial taxa by qPCR}

309 Quantitative PCR was performed to detect the above key bacterial taxa in samples from the

310 validation population, including Firmucutes, Actinobacteria, Lactobacillus, Bifidobacterium,

311 Gardnerella vaginalis, and Atopobium vaginae, and further verified the results of 16S rRNA

312 gene sequencing. The abundance of Actinobacteria, Gardnerella vaginalis, and Atopobium

313 vaginae were significantly enriched in the POI group $(P=0.001, P=0.007, P=0.010$,

314 respectively), and no statistically difference was found in Firmucutes $(P>0.05)$, in

315 accordance with the results obtained by 16S rRNA gene sequencing (Supplementary Fig. 3).

316 Lactobacillus was significantly decreased in POI patients $(P=0.006)$, and no statistically

317 difference was found in Bifidobacterium $(P>0.05)$, while the sequencing results showed that

318 Lactobacillus was decreased in POI patients but not statistically significant, and

319 Bifidobacterium was significantly decreased.

320 Analyses of correlations between vaginal microbiota and clinical indicators related to

321 ovarian reserve, ovarian endocrine function, and symptoms of perimenopausal

322 syndrome

323 The association between vaginal microbiological characteristics (alpha diversity indices,

324 relative abundance of bacterial taxa) and clinical indicators related to ovarian reserve (AMH,

325 inhibin B), ovarian endocrine function (FSH, LH, PRL, PRG, T, E2), and symptoms of

326 perimenopausal syndrome (KMI score) were investigated. No significant correlation was

327 found between alpha diversity and these clinical indicators $(P>0.05)$ (Supplementary Fig. 4),

328 as were the relative abundances of Firmucutes, Lactobacillus, and Streptococcus (Fig. 4). The

329 relative abundances of Actinobacteria, Gardnerella, and Atopobium were all significantly

330 negatively correlated with serum AMH and inhibin B levels, which were usually decreased in 
331 POI patients, while significantly positive correlations were found in Bifidobacterium. The

332 relative abundances of Actinobacteria, Gardnerella, and Atopobium were all significantly

333 positively correlated with serum FSH and LH levels, which were usually increased

334 dramatically in POI patients, while Bifidobacterium was significantly negatively correlated

335 with serum FSH levels. E2 is one of the most important steroid hormones secreted by the

336 ovaries, and its level is significantly reduced in POI patients. The relative abundance of

337 Atopobium was significantly negatively correlated with serum E2 levels, while

338 Bifidobacterium was significantly positively correlated with serum E2 levels. The KMI score

339 reflects the severity of the symptoms of perimenopausal syndrome. We found that the relative

340 abundances of Gardnerella and Atopobium were all significantly positively correlated with

341 the KMI score. Collectively, the increase of Gardnerella and Atopobium and the decrease of

342 Bifidobacterium in vaginal microbiota were significantly correlated with declined ovarian

343 reserve, endocrine disruption, and symptoms of perimenopausal syndrome in POI patients.

\section{The potential of key bacterial taxa in the vagina to predict POI}

345 For key bacterial taxa with significant differences in relative abundance between the two

346 groups, including Actinobacteria, Gardnerella, Atopobium and Bifidobacterium, ROC curves

347 were constructed and AUC values were calculated to assess their potential to predict POI. The

348 accuracies of Gardnerella and Atopobium in predicting POI were considered acceptable, the

349 corresponding AUC values were 0.732 and 0.759 , respectively, which were both greater than

$350 \quad 0.7^{49}$ (Fig. 5). However, the accuracies of Actinobacteria and Bifidobacterium were very low,

351 with AUC values of 0.685 and 0.678 , respectively, which were both less than $0.7^{49}$. Our

352 results indicated that the accuracy of a single bacterial taxa for predicting POI was limited,

353 and more other relevant indicators may need to be combined to provide improved early

354 detection of POI. 


\section{Principal findings of the study}

357 In this case-control study, with 16S rRNA gene high-throughput sequencing technology, we

358 found that the relative abundance of Actinobacteria, Atopobium, and Gardnerella were

359 significantly increased in POI patients, while Bifidobacterium was significantly decreased.

360 Moreover, these changes in vaginal microbiota were significantly correlated with declined

361 ovarian reserve, endocrine disruption, and symptoms of perimenopausal syndrome in POI

362 patients. These bacterial taxa may be potential new biomarkers for POI and related to the

363 occurrence and development of POI, but further researches and explorations are needed.

\section{Interpretation of the findings}

365 POI is highly heterogeneous in etiology and the exact etiology remains unclear. Infectious

366 factors may also be related to POI, such as mumps virus, human immunodeficiency virus

367 (HIV), varicella zoster virus, and shigella ${ }^{50}$. While, there are only case reports or

368 epidemiological data, lacking of etiological mechanism research. Due to the limitations of

369 traditional culture-based techniques, our current findings may be just the tip of the iceberg.

370 Besides, most of these reports focus on extraneous virous infection, while it is still unclear

371 whether the disturbance of organism microbiota, such as vaginal microbiota, the most

372 important microbiota of the female reproductive system, are related to POI. Under this

373 circumstance, we carried out this research, hoping to contribute to this field.

374 In recent years, a large number of researches have shown that the vaginal microbiota plays an

375 important role in maintaining the health of female reproductive system ${ }^{16,27,51-57}$. Although

376 other anaerobic and aerobic bacteria colonize the vagina, Lactobacillus is the dominant

377 species in most women ${ }^{32,58}$. The relative abundance of Lactobacillus is significantly reduced

378 in postmenopausal women ${ }^{17,59}$, and a similar change has been observed in women with 
premature ovarian failure $(\mathrm{POF})^{33}$. In our study, we also observed a decrease in Lactobacillus

380 in POI patients, but the difference was not statistically significant. With the in-depth study of

381 the etiology and the accumulation of clinical cases, doctors and researchers have gradually

382 realized that ovarian aging is a group of diseases with diverse clinical manifestations,

383 complex etiology, and progressive development, including the hidden stage, the biochemical

384 abnormal stage and the clinical abnormal stage, and menopause, POF and POI represent

385 different stages in the progression of ovarian aging ${ }^{1,37,60,61}$. POI is a disease state that occurs

386 when ovarian function declines to a certain stage before the age of 40 . To a certain extent, it

387 can reflect the diversity and progress of ovarian aging. However, menopause and POF can

388 only represent the terminal stage of ovarian failure and cannot reflect the progression of the

389 disease. In the definition of POI and POF, the diagnostic threshold of FSH is different. The

390 diagnostic criteria of POF is FSH $>40 \mathrm{IU} / \mathrm{L}$, while the diagnostic criteria of $\mathrm{POI}$ is FSH $>25$

391 IU/L. The latter lowers the diagnostic threshold of FSH in order to identify POI patients as

392 early as possible to achieve the purpose of early diagnosis and early treatment. Therefore, in

393 the following analysis, we divided the POI patients into two groups according to the level of

394 FSH and then conducted a subgroup analysis. We found that POI patients with FSH levels of

395 25-40 IU/L compared with the control group, the relative abundance of Lactobacillus was

396 lower but not statistically significant, while the relative abundance of Lactobacillus in POI

397 patients with $\mathrm{FSH} \geq 40 \mathrm{IU} / \mathrm{L}$ was significantly lower than that of the control group $(58.77 \%$

398 vs 79.54\%, $P=0.032$ ) (Supplementary Fig. 5C). Our results suggest that the abundance of

399 vaginal Lactobacillus may gradually decrease during the decline of ovarian function, and

400 further studies are needed in the future to explore whether changes in the vaginal microbiota

401 of patients are progressing gradually like ovarian aging.

402 The bacteria inhabiting the human vagina are thought to be the first line of defense against

403 vaginal infections ${ }^{20}$. Lactobacillus inhibit binding of other bacteria to epithelial cells and

404 produce substances, including lactic acid and hydrogen peroxide, that directly kill or inhibit

405 the growth of other microorganisms ${ }^{16,21,62}$. Intracellular lactic acid is an epigenetic regulator of

406 gene activity, lactic acid blocks histone deacetylases, thereby enhancing gene transcription 
and DNA repair ${ }^{63}$. Another novel property of lactic acid is its ability to induce autophagy in epithelial cells to degrade intracellular aged or defective organelles, dysfunctional proteins as well as bacteria, viruses, protozoa, and their components, and promote homeostasis ${ }^{64-66}$. More importantly, the ability of Lactobacillus to inhibit infection without inducing inflammation can even inhibit the release of proinflammatory factors when the innate immunity is actived ${ }^{21}$. However, epigenetics, autophagy and inflammation are closely related to the occurrence and development of POI, and the reduction of Lactobacillus in the vagina of POI patients may induce the occurrence of POI and accelerate the development of it through the above-mentioned mechanisms, but further in-depth studies are essential to verify this conjecture $^{38,67,68}$.

We found that the relative abundances of Atopobium and Gardnerella, which both belonged to Actinobacteria, were significantly increased in POI patients, and were significantly correlated with declined ovarian reserve, endocrine disruption, and symptoms of perimenopausal syndrome in POI patients. Whereas, subgroup analysis only found significantly differences between POI patients with FSH $\geq 40 \mathrm{IU} / \mathrm{L}$ and the control group $(15.28 \%$ vs $0.01 \%, P<0.001$; $11.08 \%$ vs $3.14 \%, P=0.002$ ) (Supplementary Fig. 5E, F). Gardnerella vaginalis and Atopobium vaginae are anaerobic bacteria, which are one of the most commom pathogens causing bacterial vaginitis. The excessive growth of Gardnerella vaginalis can promote the release of TNF- $\alpha$, IL- 6 , and IL- $8^{34-36}$. In vitro experiments have also found that it can active the NLRP3 inflammasome signaling pathway ${ }^{69}$. Atopobium vaginae has also been found to be related to gene mutations related to the TLR2 signaling pathway, which is one of the key pathways of inflammation ${ }^{70}$. The increase of Gardnerella vaginalis and Atopobium vaginae in POI patients may enhance the inflammatory response through a variety of mechanisms, leading to a chronic inflammatory state, thereby affecting the function of ovaries.

The relative abundance of Bifidobacterium was dramatically decreased in POI patients. Bifidobacterium is mainly colonized in the human intestines, and only a small amount is colonized in the vagina. As a kind of probiotic, it has the effects of anti-inflammatory, 
434 improving immune function, and resisting oxidative damage ${ }^{71-73}$. Clinical studies have found

435 that Bifidobacterium can improve the metabolism and cardiovascular function of

436 postmenopausal women ${ }^{74-76}$. Studies have also found that Bifidobacterium can regulate the

437 secretion of sex hormones in polycystic ovarian syndrome patients and significantly reduce

438 serum LH levels and $\mathrm{LH} / \mathrm{FSH}$ ratio $^{77}$. There is no research on probiotics in POI patients

439 currently. Whether probiotics such as Bifidobacterium can delay the development of POI or

440 contribute to the treatment of POI is still unknown and remains to be explored in the future.

441 Moreover, due to the influence of human internal factors and environmental factors, the

442 individual differences in the use of probiotics are relatively large. The mainstream trend in the

443 future is personalized probiotic intervention strategies. This is also true for POI patients.

444 Individualized probiotic intervention studies need to be carried out according to the patient's

445 own situation.

446 Strengths and limitations

447 The strength of our study lies in the comprehensive description of vaginal bacterial

448 communities associated with POI archived through 16S rRNA gene sequencing. We identified

449 bacterial taxa that were enriched in POI patients and evaluated their value in predicting POI.

450 Particularly, the correlations between the vaginal microbiota and the clinical characteristics,

451 mainly ovarian reserve and ovarian endocrine function indices, were analyzed. However, the

452 limitations of the current study also merit careful consideration. We only focused on changes

453 in vaginal microbiota in POI patients, but did not study the microbiota in other parts of the

454 body, such as gut, mouth, skin, etc. Furthermore, our results can only suggest that there is a

455 correlation between vaginal microbiota and POI, but the causal relationship between them is

456 still unclear, and we could not clarify detailed roles of specific constituents of the vaginal

457 microbiota in the pathogenesis of POI. Therefore, future studies involving larger and wider

458 study populations, prospective cohort, and animal models are needed to more

459 comprehensively describe the composition of vaginal microbiota under different stages of

460 ovarian aging and explore potential mechanisms underlying the association of the vaginal 
461 microbiota and POI. Researches on the gut microbiota of POI patients may also help to clarify

462 the role of microbiota in POI and improve many aspects of prevention and treatment.

\section{Conclusions}

464 In summary, our study found that the relative abundance of Actinobacteria, Atopobium, and

465 Gardnerella were significantly increased in POI patients, while Bifidobacterium was

466 significantly decreased. Moreover, these changes in vaginal microbiota were significantly

467 correlated with declined ovarian reserve, endocrine disruption, and symptoms of

468 perimenopausal syndrome in POI patients. Our study revealed the correlation between vaginal

469 microbiota and POI, filled the gap in the field of microbial and POI research and provided a

470 new research strategy for POI. But the causal relationship is still unclear, and we could not

471 clarify detailed roles of specific constituents of the vaginal microbiota in the pathogenesis of

472 POI. Therefore, future studies are needed to explore potential mechanisms underlying the

473 association of vaginal microbiota and POI.

$474 \quad$ Additional files

475 Additional file 1: Table S1. Primer sequences for quantitative PCR.

476 Additional file 2: Table S2. Clinical information of participants whose samples were used for

477 16S rRNA gene sequencing (demographics, lifestyle, menstrual and reproductive history,

478 HAD score).

479 Additional file 3: Table S3. Phylum abundance.

480 Additional file 4: Table S4. Genus abundance.

481 Additional file 5: Table S5. Species abundance. 
Additional file 6: Table S6. Dominant bacterial taxa and its abundance in each sample.

483 Additional file 7: Figure S1. Comparison of vaginal microbiota diversity of women with

484 POI and healthy controls. A-E. Comparison of alpha diversity index Observed-otus, Chao 1

485 index, Shannon index, Simpson index and Goods_coverage. F. Beta diversity was

486 significantly different between the two groups according to the Anosim (analysis of

487 similarities, $\mathrm{R}=0.083, \mathrm{P}=0.01$ ) and showed in PCA (principal component analysis) plots.

488 Additional file 8: Figure S2. Composition of the vaginal microbiota of POI patients and the 489 controls. Relative abundance was shown at the phylum (A), class (B), order (C), family (D), 490 genus (E) and species (F) levels.

491 Additional file 9: Figure S3. qPCR was used to validate the key bacterial taxa in samples 492 from the validation population. $P<0.05$ was considered as statistically significant. ${ }^{*} P<0.05$, $493 \quad{ }^{* *} P<0.01$.

494 Additional file 10: Figure S4. No spearman correlation was found between alpha diversity 495 indices of vaginal microbiota and clinical indicators related to ovarian reserve, ovarian 496 endocrine function, and symptoms of perimenopausal syndrome. AMH, anti-mullerian 497 hormone; FSH, follicle stimulating hormone; LH, luteinizing hormone; PRL, prolactin; PRG, 498 progesterone; T, testosterone; E2, estradiol.

499 Additional file 11: Figure S5. Subgroup comparison of the relative abundance of vaginal 500 microbiota based on serum FSH levels. $P<0.05$ was considered as statistically significant.

$501{ }^{*}$ Comparison between POI patients with FSH levels of 25-40 IU/L and the control group, ${ }^{*} P$ $502<0.05,{ }^{* *} P<0.01$. ${ }^{\#}$ Comparison between POI patients with FSH $\geq 40 \mathrm{IU} / \mathrm{L}$ and the control 503 group, ${ }^{\#} P<0.05,{ }^{\#} P<0.01$. FSH, follicle stimulating hormone. 
505 POI: premature ovarian insufficiency; HRT: hormone replacement therapy; IVF-ET: in vitro

506 fertilization-embryo transfer; HIV: human immunodeficiency virus; TNF: tumor necrosis

507 factor; IL: interleukin; FSH: follicle stimulating hormone; KMI: Kupperman index; HAD:

508 hospital anxiety and depression; qPCR: quantitative polymerasechain reaction; LEfSe: linear

509 discriminant analysis effect size; SD: standard deviation; IQR: interquartile; ROC: received

510 operating characteristic; AUC: area under the curve; BMI: body mass index; LH: luteinizing

511 hormone; T: testosterone; E2: estradiol; AMH: anti-mullerian hormone; PRL: prolactin; PRG:

512 progesterone; PCA: principal component analysis; POF: premature ovarian failure.

\section{Acknowledgements}

514 We thank all the participants who were involved in the study, as well as the doctors, nurses

515 and the laboratory staff who helped with the study.

\section{Funding}

517 The study was supported by the grant from the Clinical Research Pilot Project of Tongji

518 hospital, Huazhong University of Science and Technology (No. 2019CR205), and the

519 National Natural Science Foundation of China (NO. 81671394, NO.81701438, and

$520 \quad$ NO.82001498).

\section{$521 \quad$ Availability of data and materials}

522 Raw sequence data files for the samples described in this study were deposited to the NCBI

523 Sequence Read Archive. The data that support the findings of this study are available from the 524 corresponding authors upon reasonable request. 
526 WJY, ZJJ and WSX conceived and designed the study. WJY, FYZ, YW, YSZ and LAY

527 recruited participants and collected their vaginal samples and related information. WJY and

528 ZJJ analyzed the data. WJY and WSX drafted and revised the paper. All authors reviewed the

529 results and approved the final version of the manuscript.

\section{Competing interest}

531 The authors declare that they have no competing interests.

\section{Ethics approval}

533 This study and all its protocol were approved by the Ethical Committee of Tongji Hospital,

534 Tongji Medical College, Huazhong University of Science and Technology. All participants

535 agreed to join the study and signed an informed consent form.

\section{Author details}

$537{ }^{1}$ Department of Obstetrics and Gynecology, Tongji Hospital, Tongji Medical College,

538 Huazhong University of Science and Technology, 1095 Jiefang Avenue, Wuhan, Hubei,

539 China.

$540 \quad{ }^{2}$ National Clinical Research Center for Obstetrical and Gynecological Diseases, Tongji

541 Hospital, Tongji Medical College, Huazhong University of Science and Technology, 1095

542 Jiefang Avenue, Wuhan, Hubei, China. 
$543{ }^{3}$ Cancer Biology Research Center, Key Laboratory of the Ministry of Education, Tongji

544 Hospital, Tongji Medical College, Huazhong University of Science and Technology, 1095

545 Jiefang Avenue, Wuhan, Hubei, China.

\section{References}

548 1. European Society for Human R, Embryology Guideline Group on POI, Webber L, et 549 al. ESHRE Guideline: management of women with premature ovarian insufficiency.

$550 \quad$ Hum Reprod. 2016;31(5):926-937.

551 2. Tucker EJ, Grover SR, Bachelot A, Touraine P, Sinclair AH. Premature Ovarian

552 Insufficiency: New Perspectives on Genetic Cause and Phenotypic Spectrum. Endocr 553 Rev. 2016;37(6):609-635.

554 3. Domniz N, Meirow D. Premature ovarian insufficiency and autoimmune diseases.

555 Best Pract Res Clin Obstet Gynaecol. 2019;60:42-55.

556 4. Christ JP, Gunning MN, Palla G, et al. Estrogen deprivation and cardiovascular

557 disease risk in primary ovarian insufficiency. Fertility and Sterility.

558 2018;109(4):594-600.e591.

559 5. Popat VB CK, Vanderhoof VH, et al. Bone mineral density in estrogen-deficient

$560 \quad$ young women. J Clin Endocrinol Metab 2009;9:2277-2283.

561 6. Schmidt PJ, Luff JA, Haq NA, et al. Depression in women with spontaneous 46, XX

562 primary ovarian insufficiency. J Clin Endocrinol Metab. 2011;96(2):E278-287.

563 7. Qin Y, Jiao X, Simpson JL, Chen ZJ. Genetics of primary ovarian insufficiency: new 564 developments and opportunities. Hum Reprod Update. 2015;21(6):787-808.

565 8. Huhtaniemi I, Hovatta O, La Marca A, et al. Advances in the Molecular Pathophysiology, Genetics, and Treatment of Primary Ovarian Insufficiency. Trends

9. Jiao X, Ke H, Qin Y, Chen ZJ. Molecular Genetics of Premature Ovarian

Insufficiency. Trends in endocrinology and metabolism: TEM. 2018;29(11):795-807.

10. Silva CA, Yamakami LY, Aikawa NE, Araujo DB, Carvalho JF, Bonfa E. Autoimmune primary ovarian insufficiency. Autoimmunity reviews. 2014;13(4-5):427-430.

11. Spears N, Lopes F, Stefansdottir A, et al. Ovarian damage from chemotherapy and current approaches to its protection. Human reproduction update. 2019;25(6):673-693.

12. Gracia CR, Sammel MD, Freeman E, et al. Impact of cancer therapies on ovarian reserve. Fertil Steril. 2012;97(1):134-140 e131. 
13. Zhao H, Jin L, Li Y, et al. Oncofertility: What can we do from bench to bedside? Cancer Lett. 2019;442:148-160.

14. Vabre P, Gatimel N, Moreau J, et al. Environmental pollutants, a possible etiology for premature ovarian insufficiency: a narrative review of animal and human data. Environ Health. 2017;16(1):37.

15. Li C, Cao M, Ma L, et al. Pyrethroid Pesticide Exposure and Risk of Primary Ovarian Insufficiency in Chinese Women. Environ Sci Technol. 2018;52(5):3240-3248.

16. Ma B, Forney LJ, Ravel J. Vaginal Microbiome: Rethinking Health and Disease. Annual Review of Microbiology. 2012;66(1):371-389.

17. Ouarabi L, Drider D, Taminiau B, Daube G, Bendali F, Lucau-Danila A. Vaginal Microbiota: Age Dynamic and Ethnic Particularities of Algerian Women. Microbial ecology. 2020.

18. Wang S, Ryan CA, Boyaval P, Dempsey EM, Ross RP, Stanton C. Maternal Vertical Transmission Affecting Early-life Microbiota Development. Trends in microbiology. 2020;28(1):28-45.

19. McDonald B, McCoy KD. Maternal microbiota in pregnancy and early life. Science. 2019;365(6457):984-985.

20. Lewis FMT, Bernstein KT, Aral SO. Vaginal Microbiome and Its Relationship to Behavior, Sexual Health, and Sexually Transmitted Diseases. Obstetrics \& Gynecology. 2017;129(4):643-654.

21. Aldunate M, Srbinovski D, Hearps AC, et al. Antimicrobial and immune modulatory effects of lactic acid and short chain fatty acids produced by vaginal microbiota associated with eubiosis and bacterial vaginosis. Front Physiol. 2015;6:164.

22. Ness RB, Hillier SL, Kip KE, et al. Bacterial vaginosis and risk of pelvic inflammatory disease. Obstet Gynecol. 2004;104(4):761-769.

23. Nelson DB HA, Wu G, et al. First trimester levels of BV-associated bacteria and risk of miscarriage among women early in pregnancy. Matern Child Health J. 2015;19:2682-2687.

24. DiGiulio DB, Callahan BJ, McMurdie PJ, et al. Temporal and spatial variation of the human microbiota during pregnancy. Proc Natl Acad Sci U S A.

2015;112(35):11060-11065.

25. Freitas AC, Bocking A, Hill JE, Money DM. Increased richness and diversity of the

26. Ravel J GP, Abdo Z, Schneider GM, Koenig SS, McCulle SL, Karlebach S, Gorle R, vaginal microbiota and spontaneous preterm birth. Microbiome. 2018;6(1):117.

Russell J, Tacket CO. Vaginal microbiome of reproductive age women. Proc Natl Acad Sci U S A. 2011;108(Suppl 1):4680-4687.

27. Martin DH, Marrazzo JM. The Vaginal Microbiome: Current Understanding and Future Directions. The Journal of infectious diseases. 2016;214 Suppl 1:S36-41.

28. Romero R. The composition and stability of the vaginal microbiota of normal

29. Chaban B. Characterization of the vaginal microbiota of healthy Canadian women through the menstrual cycle. Microbiome. 2014. 
30. Gliniewicz K, Schneider GM, Ridenhour BJ, et al. Comparison of the Vaginal Microbiomes of Premenopausal and Postmenopausal Women. Frontiers in microbiology. 2019;10:193.

31. Kaur H, Merchant M, Haque MM, Mande SS. Crosstalk Between Female Gonadal Hormones and Vaginal Microbiota Across Various Phases of Women's Gynecological Lifecycle. Frontiers in microbiology. 2020;11:551.

32. Muhleisen AL, Herbst-Kralovetz MM. Menopause and the vaginal microbiome. Maturitas. 2016;91:42-50.

33. Wang J, Xu J, Han Q, et al. Changes in the vaginal microbiota associated with primary ovarian failure. BMC Microbiol. 2020;20(1):230.

34. Jang SE, Jeong JJ, Choi SY, Kim H, Han MJ, Kim DH. Lactobacillus rhamnosus HN001 and Lactobacillus acidophilus La-14 Attenuate Gardnerella vaginalis-Infected Bacterial Vaginosis in Mice. Nutrients. 2017;9(6).

35. Shishpal P, Kasarpalkar N, Singh D, Bhor VM. Characterization of Gardnerella vaginalis membrane vesicles reveals a role in inducing cytotoxicity in vaginal epithelial cells. Anaerobe. 2020;61:102090.

36. Wong YP, Tan GC, Wong KK, Anushia S, Cheah FC. Gardnerella vaginalis in perinatology: An overview of the clinicopathological correlation. The Malaysian journal of pathology. 2018;40(3):267-286.

37. Davis SR LI, Lumsden M, et al. Menopause. Nat Rev Dis Primers. 2015;1:15004.

38. Huang Y, Hu C, Ye H, et al. Inflamm-Aging: A New Mechanism Affecting Premature Ovarian Insufficiency. Journal of immunology research. 2019;2019:8069898.

39. Yang H PH, Miao CX. Ovarian IL-1 $\alpha$ and IL-1 $\beta$ levels are associated with primary ovarian insufficiency. Int J Clin Exp Pathol. 2018;11(9):4711-4717.

40. Sun S, Chen H, Zheng X, Ma C, Yue R. Analysis on the level of IL-6, IL-21, AMH in patients with auto-immunity premature ovarian failure and study of correlation. Exp Ther Med. 2018;16(4):3395-3398.

41. Logue JB, Stedmon CA, Kellerman AM, et al. Experimental insights into the importance of aquatic bacterial community composition to the degradation of dissolved organic matter. Isme j. 2016;10(3):533-545.

42. Magoč T, Salzberg SL. FLASH: fast length adjustment of short reads to improve genome assemblies. Bioinformatics. 2011;27(21):2957-2963.

43. Rognes T, Flouri T, Nichols B, Quince C, Mahé F. VSEARCH: a versatile open source tool for metagenomics. PeerJ. 2016;4:e2584.

44. Callahan BJ, McMurdie PJ, Rosen MJ, Han AW, Johnson AJ, Holmes SP. DADA2: High-resolution sample inference from Illumina amplicon data. Nat Methods. 2016;13(7):581-583.

45. Blaxter M, Mann J, Chapman T, et al. Defining operational taxonomic units using DNA barcode data. Philos Trans R Soc Lond B Biol Sci. 2005;360(1462):1935-1943.

46. Bolyen E, Rideout JR, Dillon MR, et al. Reproducible, interactive, scalable and extensible microbiome data science using QIIME 2. Nature biotechnology. 2019;37(8):852-857. 
47. Tao M, Shao H, Li C, Teng Y. Correlation between the modified Kupperman Index and the Menopause Rating Scale in Chinese women. Patient preference and adherence. 2013;7:223-229.

48. Zigmond AS, Snaith RP. The hospital anxiety and depression scale. Acta psychiatrica Scandinavica. 1983;67(6):361-370.

49. JN M. Receiver Operating Characteristic Curve in Diagnostic Test Assessment. Journal of thoracic oncology : official publication of the International Association for the Study of Lung Cancer. 2010;5(9):1315-1316.

50. De Vos M, Devroey P, Fauser BC. Primary ovarian insufficiency. Lancet.

51. White BA, Creedon DJ, Nelson KE, Wilson BA. The vaginal microbiome in health and disease. Trends in endocrinology and metabolism: TEM. 2011;22(10):389-393.

52. Franasiak JM, Scott RT, Jr. Introduction: Microbiome in human reproduction. Fertility and sterility. 2015;104(6):1341-1343.

53. Green KA, Zarek SM, Catherino WH. Gynecologic health and disease in relation to the microbiome of the female reproductive tract. Fertility and sterility. 2015;104(6):1351-1357.

54. Smith SB, Ravel J. The vaginal microbiota, host defence and reproductive physiology.

55. Younes JA, Lievens E, Hummelen R, van der Westen R, Reid G, Petrova MI. Women

56. Greenbaum S, Greenbaum G, Moran-Gilad J, Weintraub AY. Ecological dynamics of the vaginal microbiome in relation to health and disease. American journal of obstetrics and gynecology. 2019;220(4):324-335.

57. Koedooder R, Mackens S, Budding A, et al. Identification and evaluation of the microbiome in the female and male reproductive tracts. Human reproduction update. 2019;25(3):298-325.

58. Martin DH. The microbiota of the vagina and its influence on women's health and

59. Pabich WL FS, Stamm WE,Scholes D,Boyko EJ,Gupta K. Prevalence and determinants of vaginal flora alterations in postmenopausal women. The Journal of infectious diseases. 2003;188(7):1054-1058.

60. Laisk T, Tsuiko O, Jatsenko T, et al. Demographic and evolutionary trends in ovarian function and aging. Human reproduction update. 2019;25(1):34-50.

61. Shelling AN. Premature ovarian failure. Reproduction (Cambridge, England). 2010;140(5):633-641.

62. Witkin SS, Linhares IM. Why do lactobacilli dominate the human vaginal microbiota? BJOG : an international journal of obstetrics and gynaecology. 2017;124(4):606-611.

63. Latham T, Mackay L, Sproul D, et al. Lactate, a product of glycolytic metabolism, inhibits histone deacetylase activity and promotes changes in gene expression. Nucleic acids research. 2012;40(11):4794-4803. 
64. Ghadimi D, de Vrese M, Heller KJ, Schrezenmeir J. Lactic acid bacteria enhance autophagic ability of mononuclear phagocytes by increasing Th1 autophagy-promoting cytokine (IFN-gamma) and nitric oxide (NO) levels and reducing Th2 autophagy-restraining cytokines (IL-4 and IL-13) in response to Mycobacterium tuberculosis antigen. International immunopharmacology. 2010;10(6):694-706.

65. Wang CW, Klionsky DJ. The molecular mechanism of autophagy. Molecular medicine (Cambridge, Mass). 2003;9(3-4):65-76.

66. Dikic I, Elazar Z. Mechanism and medical implications of mammalian autophagy. Nature reviews Molecular cell biology. 2018;19(6):349-364.

67. Jackson-Cook C. A hypothesis: Could telomere length and/or epigenetic alterations contribute to infertility in females with Turner syndrome? American journal of medical genetics Part C, Seminars in medical genetics. 2019;181(1):108-116.

68. Shao T, Ke H, Liu R, Zhao S, Qin Y. Variation analysis of theTMEM150B gene in Chinese women with premature ovarian insufficiency. Reprod Biomed Online. 2019;38(3):407-412.

69. Vick EJ, Park HS, Huff KA, Brooks KM, Farone AL, Farone MB. Gardnerella vaginalis triggers NLRP3 inflammasome recruitment in THP-1 monocytes. Journal of reproductive immunology. 2014;106:67-75.

70. Taylor BD, Totten PA, Astete SG, et al. Toll-like receptor variants and cervical Atopobium vaginae infection in women with pelvic inflammatory disease. American journal of reproductive immunology. 2018;79(2).

71. Hill C, Guarner F, Reid G, et al. Expert consensus document. The International Scientific Association for Probiotics and Prebiotics consensus statement on the scope and appropriate use of the term probiotic. Nature reviews Gastroenterology \& hepatology. 2014;11(8):506-514.

72. Nowak A, Paliwoda A, Blasiak J. Anti-proliferative, pro-apoptotic and anti-oxidative activity of Lactobacillus and Bifidobacterium strains: A review of mechanisms and therapeutic perspectives. Crit Rev Food Sci Nutr. 2019;59(21):3456-3467.

73. Bernini LJ, Simao ANC, de Souza CHB, et al. Effect of Bifidobacterium lactis HN019 on inflammatory markers and oxidative stress in subjects with and without the metabolic syndrome. The British journal of nutrition. 2018:1-8.

74. Szulinska M LI, Skrypnik K et al. Multispecies Probiotic Supplementation Favorably AffectsVascular Function and ReducesArterial Stiffness inObese PostmenopausalWomen-A12-Week Placebo-Controlled and Randomized Clinical Study. Nutrients. 2018;10(11): E1672.

75. Szulinska M LI, van Hemert S et al. Dose-Dependent Effects of Multispecies Probiotic Supplementation on the Lipopolysaccharide (LPS) Level and Cardiometabolic Profile in Obese PostmenopausalWomen: A 12-Week Randomized Clinical Trial. Nutrients. 2018;10(6):E773. 
743

744

745

746

747

748

749

750

751

752

753

754

755

756

757

758

759

760
76. Barreto FM CSA, Morimoto HK et al. Beneficial effects of Lactobacillus plantarum on glycemia and homocysteine levels in postmenopausal women with metabolic syndrome. Nutrition. 2014;30(7-8):939-942.

77. Zhang J, Sun Z, Jiang S, et al. Probiotic Bifidobacterium lactis V9 Regulates the Secretion of Sex Hormones in Polycystic Ovary Syndrome Patients through the Gut-Brain Axis. mSystems. 2019;4(2). 
762 Figure 1. Comparison of vaginal microbiota composition of women with POI and healthy 763 controls. A. Sankey plot showed the top five bacterial genera in relative abundance in all 764 samples, the width of the line indicated the relative abundance of the corresponding bacteria 765 in each group. B, C. Differences in relative abundance at phylum and genus levels. $P<0.05$ 766 was considered as statistically significant. ${ }^{*} P<0.05,{ }^{* *} P<0.01$.

767 Figure 2. Comparison of dominant vaginal bacterial taxa. A. Cluster analysis of vaginal 768 bacterial communities found in 55 participants. B. Number of women in cluster A-F in the 769 two groups.

770 Figure 3. Comparison of vaginal microbiota phylotype between groups. A. Histogram of the

771 LDA scores for differentially abundant bacterial taxa. B. Taxonomic representation of 772 statistically and biologically consistent differences between the two groups displayed by a 773 cladogram. LDA, linear discriminant analysis.

774 Figure 4. Spearman correlation between the relative abundance of vaginal microbiota and 775 clinical indicators related to ovarian reserve, ovarian endocrine function, and symptoms of 776 perimenopausal syndrome. $\mathrm{AMH}$, anti-mullerian hormone; $\mathrm{FSH}$, follicle stimulating hormone; 777 LH, luteinizing hormone; PRL, prolactin; PRG, progesterone; T, testosterone; E2, estradiol. $P$ $778<0.05$ was considered as statistically significant. ${ }^{*} P<0.05,{ }^{* *} P<0.01$.

779 Figure 5. ROC curves were constructed and AUC values were calculated to assess the 780 potential of key bacterial taxa to predict POI. A. The AUC was 0.685 for Actinobacteria, $781 P=0.019$. B. The AUC was 0.732 for Gardnerella, $P=0.003$. C. The AUC was 0.759 for 782 Atopobium, $P=0.001$. D. The AUC was 0.678 for Bifidobacterium, $P=0.023$. ROC, received 783 operating characteristic; AUC, area under the curve. 


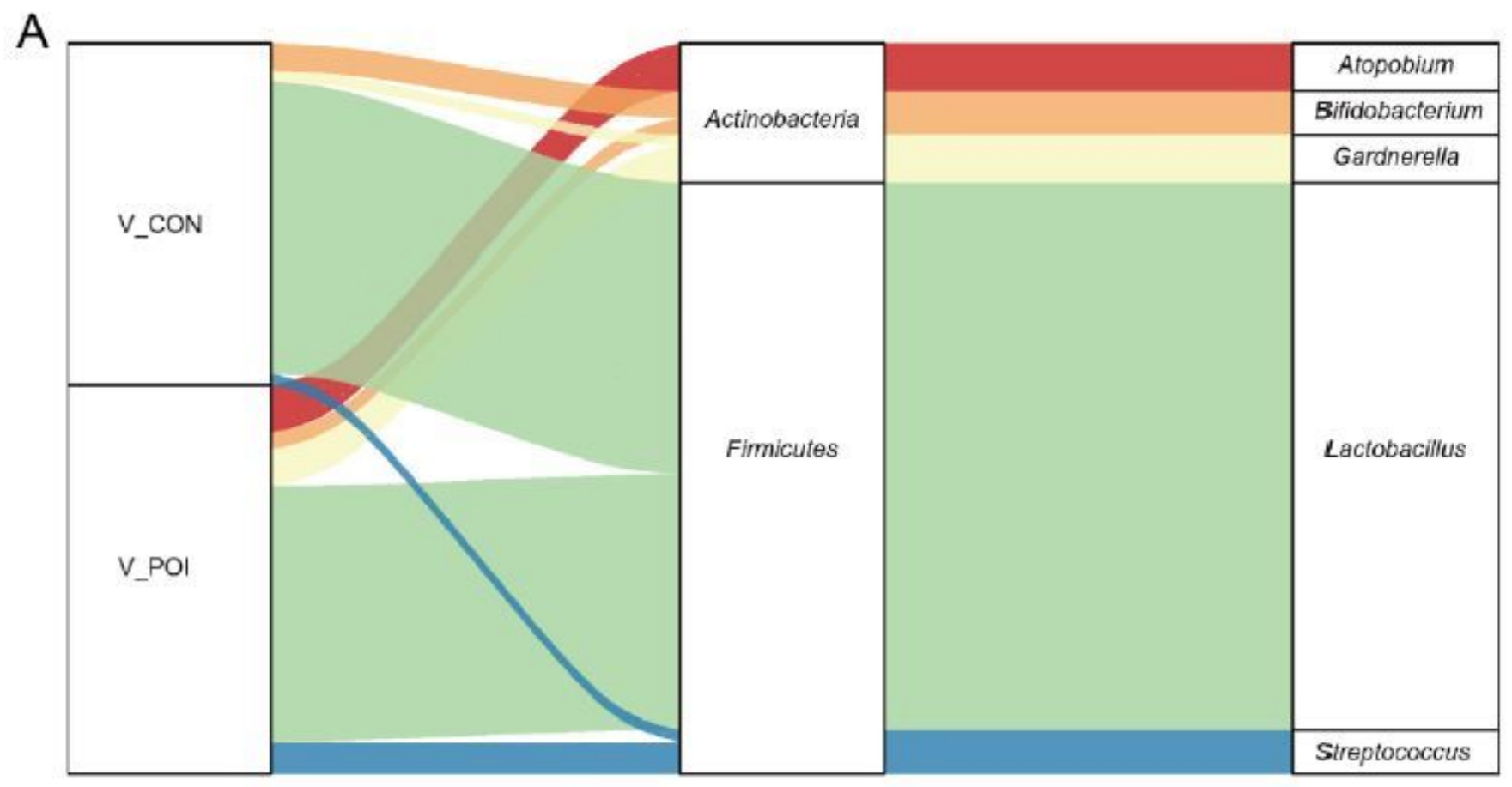

B



C

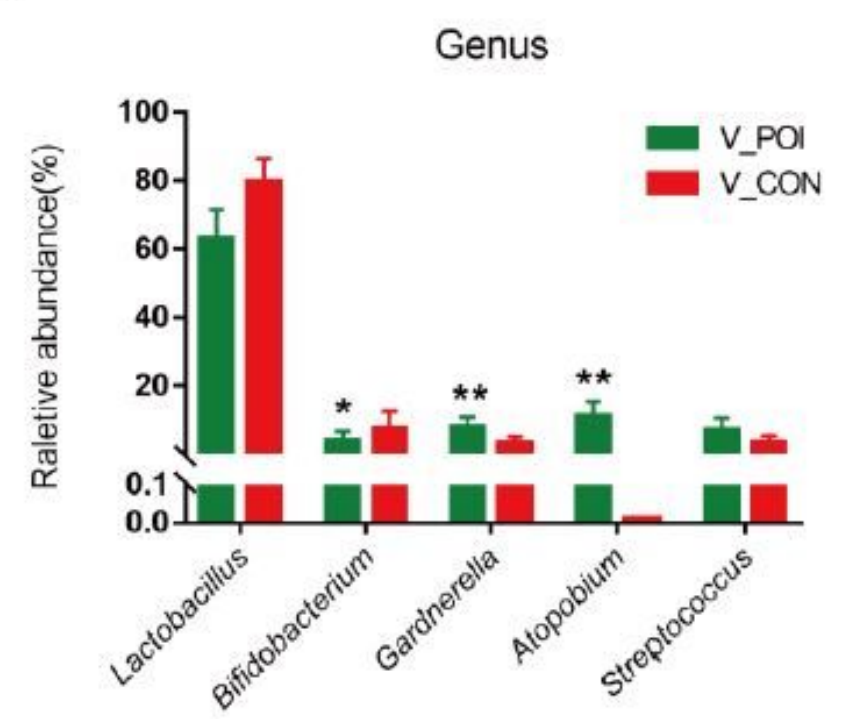

\section{Figure 1}

Comparison of vaginal microbiota composition of women with POI and healthy controls A. Sankey plot showed the top five bacterial genera in relative abundance in all samples, the width of the line indicated the relative abundance of the corresponding bacteria in each group. B , C. Differences in relative abundance at phylum and genus level s . P $\otimes 0.05$ was considered as statistically significant. $P \otimes 0.05, P$ $\otimes 0.01$. 
A

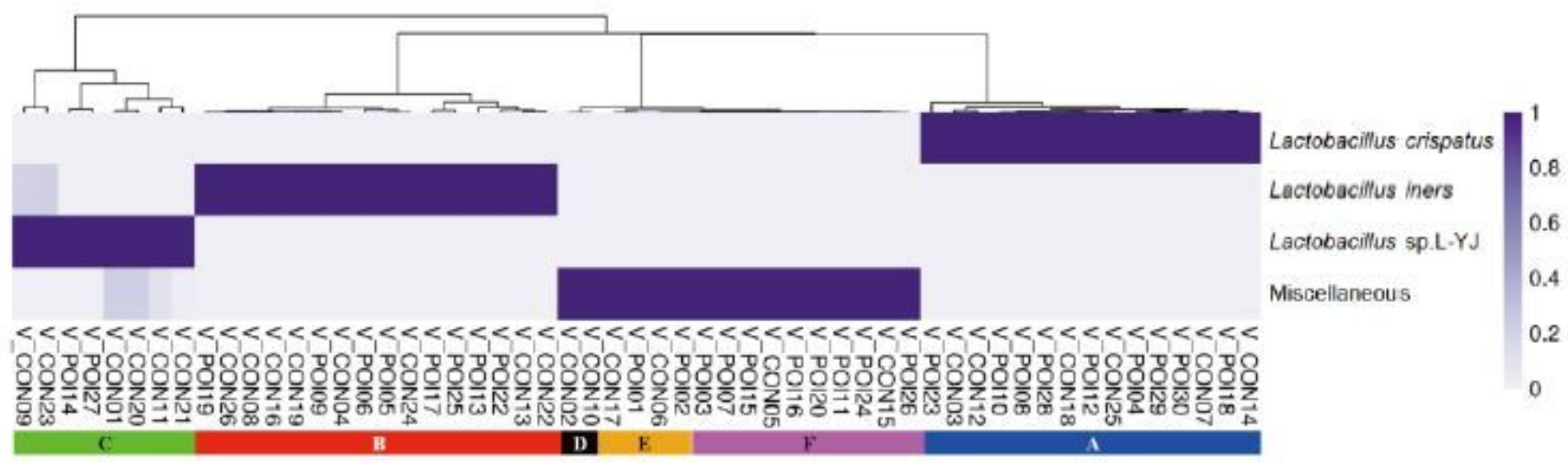

B

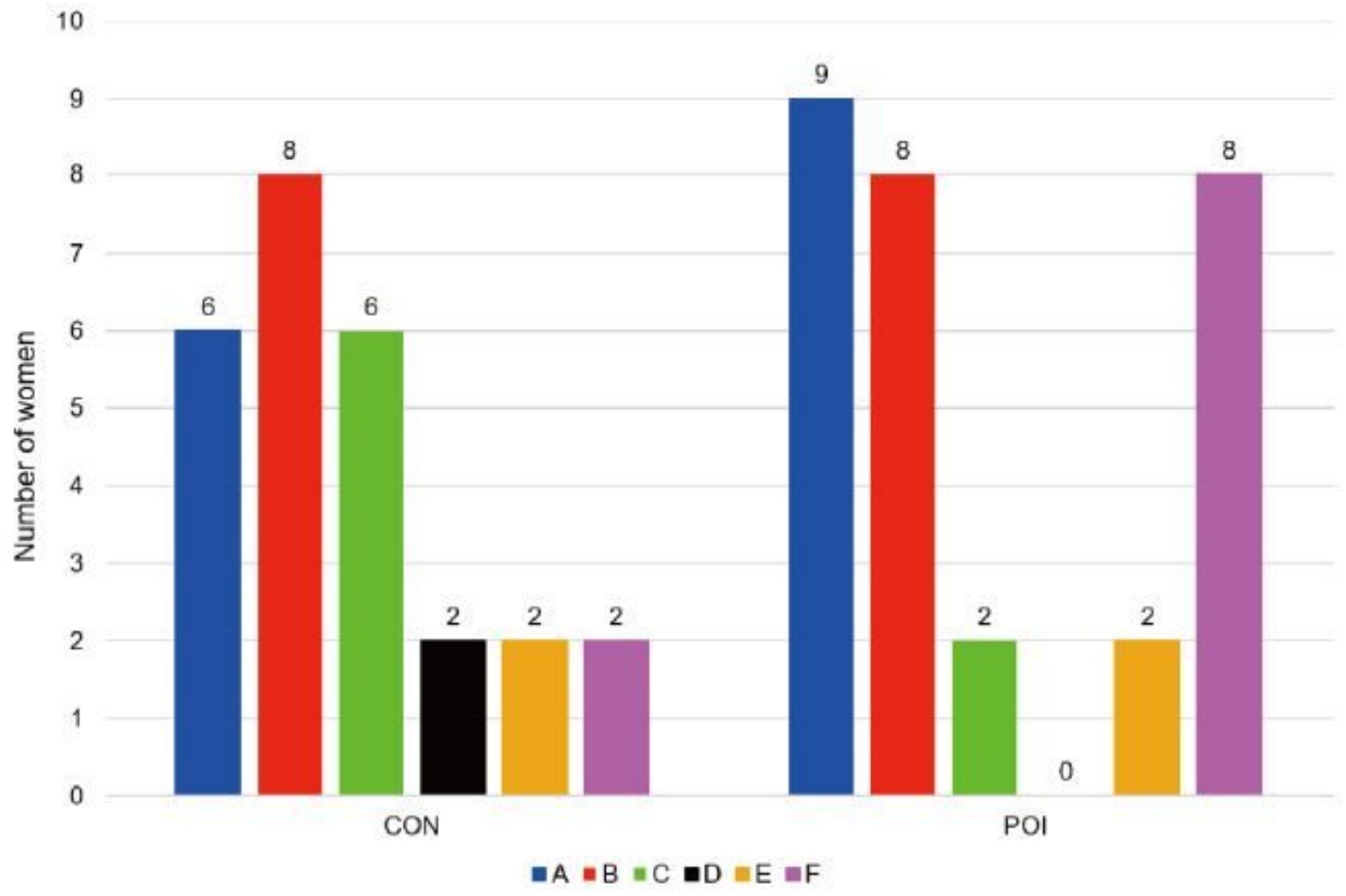

Figure 2

Comparison of dominant vagi nal bacterial taxa. A. Cluster analysis of vaginal bacterial communities found in 55 participants B. Number of women in cluster A F in the two groups. 
A

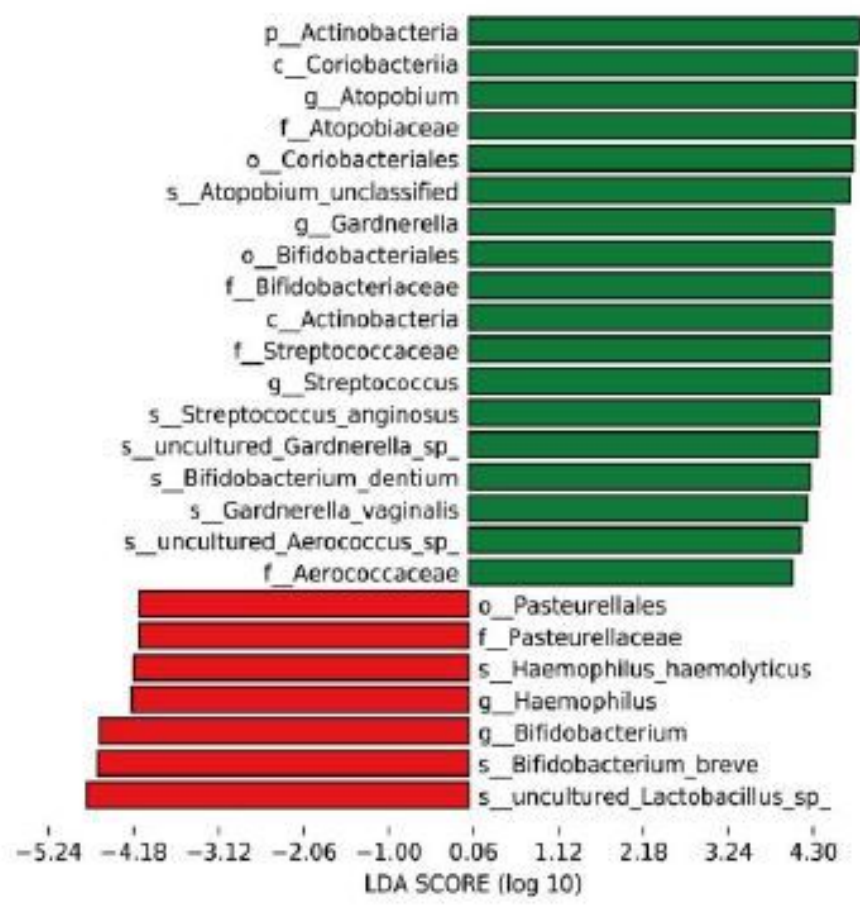

B

Cladogram

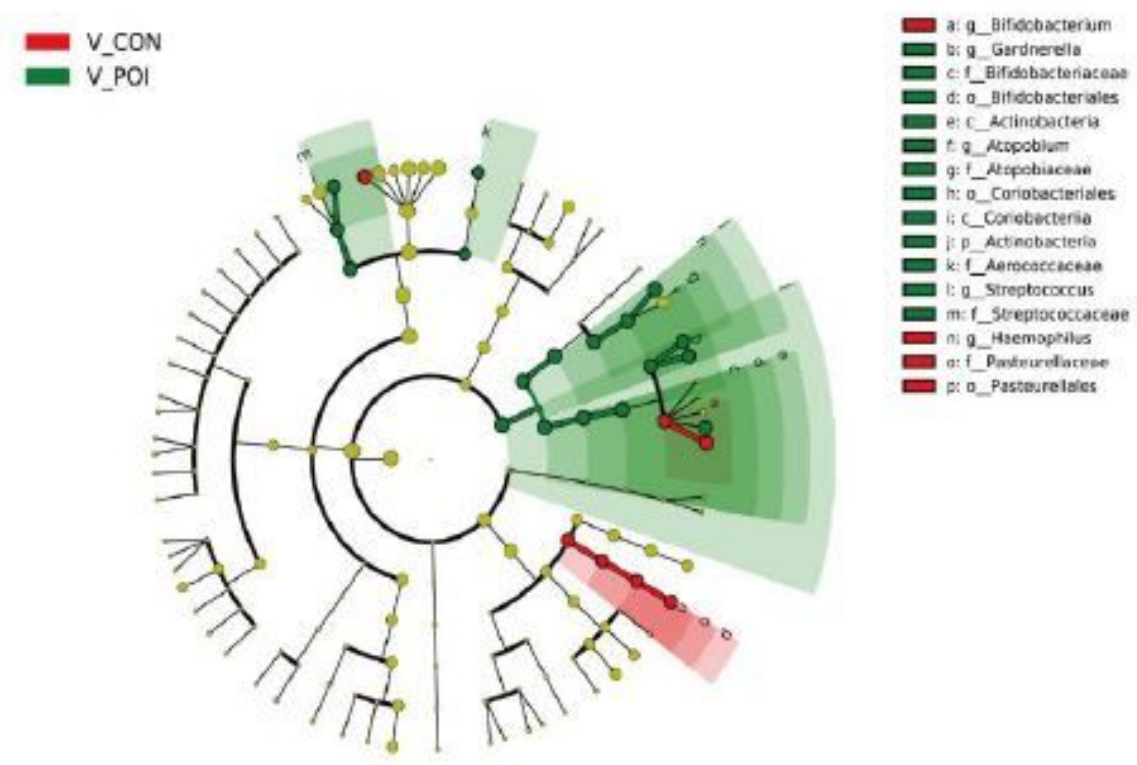

Figure 3

Comparison of vaginal microbiota phylotype between groups. A. Histogram of the LDA scores for differentially abundant bacterial taxa. B. Taxonomic representation of statistically and biologically consistent differences between the two groups displayed by a cladogram. L D A, linear discriminant analysis 


\begin{tabular}{|c|c|c|c|c|c|c|c|c|c|}
\hline 0.24 & 0.19 & -0.23 & -0.22 & 0.10 & 0.01 & -0.08 & 0.11 & -0.19 & Firmicutes \\
\hline$-0.44^{\star \star \star}$ & $-0.34^{*}$ & $0.31^{\star}$ & $0.37^{\star \star}$ & -0.15 & -0.16 & 0.01 & -0.11 & 0.26 & Actinobacteria \\
\hline 0.25 & 0.21 & -0.26 & -0.24 & 0.06 & -0.04 & -0.02 & 0.14 & -0.22 & Lactabacillus \\
\hline $0.31^{*}$ & $0.39^{\star \star}$ & $-0.40^{* \star}$ & -0.26 & 0.07 & 0.25 & 0.21 & $0.43^{\star \star}$ & -0.26 & Bifidobacterium \\
\hline$-0.49^{\star \star}$ & $-0.33^{*}$ & $0.34^{*}$ & $0.36^{* *}$ & $-0.36^{* \star}$ & -0.16 & -0.02 & -0.10 & $0.31^{*}$ & Gardnerella \\
\hline$-0.53^{* *}$ & $-0.51^{* *}$ & $0.50^{* *}$ & $0.49^{* \star}$ & -0.16 & -0.21 & -0.14 & $-0.28^{*}$ & $0.41^{\star *}$ & Atopobium \\
\hline-0.07 & -0.07 & 0.15 & 0.08 & -0.24 & 0.15 & 0.06 & -0.06 & -0.00 & Streptococcus \\
\hline  & 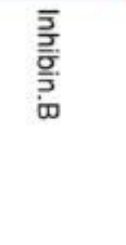 & 备 & 도 & 몸 & 중 & $\dashv$ & 思 &  & \\
\hline
\end{tabular}

\section{Figure 4}

Spearman correlation between the relative abundance of vaginal microbiota and clinical indicators related to ovarian reserve, ovarian endocrine function and symptoms of perimenopausal syndrome. AMH, antimullerian hormone; $\mathrm{FSH}$, follicle stimulating hormone; $\mathrm{LH}$, luteinizing hormone; PRL, prolactin; PRG, progesterone; T, testosterone; E2, estradiol. PP777 $\otimes 0.05$ was considered as statistically significant. P $\otimes$ $0.05, P \otimes 0.01$. 

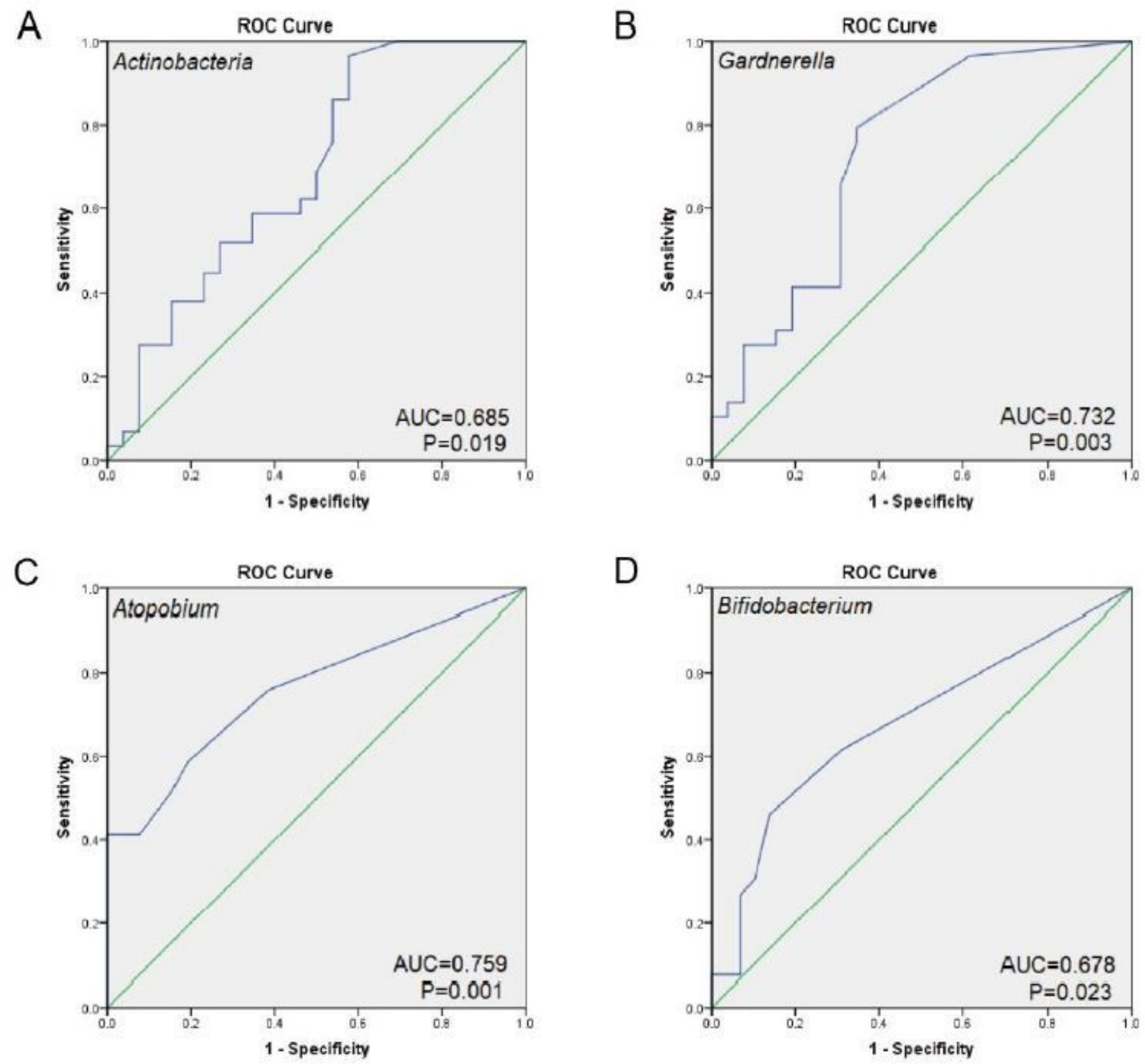

\section{Figure 5}

ROC curves were constructed and AUC values were calculated to assess the potential of key bacterial taxa to predict POI. A. T he AUC was 0.685 for Actinobact eria , $P=0.019$. B. T h e AUC was 0.732 for Gardnerella $P=0.003 \mathrm{C}$. T he AUC was 0.759 for Atopobium , $P=0.001$ D. T he AUC was 0.678 for Bifidobacterium , $P=0.023 \mathrm{ROC}$, received operating characteristic; AUC , area under the curve

\section{Supplementary Files}

This is a list of supplementary files associated with this preprint. Click to download. 
- Supplementarytable1.doc

- Supplementarytable2.doc

- Figures.pdf

- Supplementarytable3Phylumabundance.xlsx

- Supplementarytable4Genusabundance.xlsx

- Supplementarytable5Speciesabundance.xIsx

- Supplementarytable6Dominantbacterialtaxa.xlsx 